\title{
Der Grundsatz der Trennung von Staat und Religion in Japan - Zur Entscheidung des Landgerichts Osaka vom 24. März $1982^{1}$
}

\author{
von Robert Heuser
}

Am 24. März 1982 hat das LG Osaka (als erste Instanz) entschieden, daß die Umsetzung eines Kriegerdenkmals auf Kosten der Stadtgemeinde Minō (Präfektur Osaka), die kostenlose Bereitstellung eines städtischen Grundstücks für die Aufstellung des Denkmals und der Kauf dieses Grundstücks aus öffentlichen Mitteln gegen die Verfassung verstoßen haben. Die beklagten Gemeindebeamten wurden zur Kostenerstattung verurteilt. Sie haben gegen das Urteil Berufung beim Oberlandesgericht eingelegt. Eine Revision zum Obersten Gerichtshof $(\mathrm{OGH})$ ist denkbar, weswegen ein Urteil in dieser auch und besonders in Japan einem "ideologisch verminten Bereich “² zugehörigen Sache vielleicht erst Ende dieses Jahrzehnts rechtskräftig werden wird. Da die Wirkungen der Entscheidung des LG aber schon jetzt ihre Schatten werfen, ${ }^{3}$ und die Angelegenheit von grundsätzlicher Bedeutung ist für die japanische Diskussion des Verfassungsprinzips der Trennung von Staat und Religion (seikyō bunri) und damit für Selbstverständnis und Entwicklungsrichtung der gegenwärtigen japanischen Gesellschaft überhaupt, soll schon jetzt auf sie hingewiesen werden.

\section{Die Fakten}

Im Jahre 1975 plante die Stadt Minō auf dem Gelände einer städtischen Grundschule den Bau einer Schwimmhalle. Zu diesem Zweck mußte das sich dort von 1916 bis 1947 und wieder seit 1952 befindliche Kriegerdenkmal entfernt werden. Derartige aus wuchtigem Felsgestein und aufgesetzter Steinplatte mit eingemeißelter Aufschrift "chūkonhi" ("Denkmal für die loyalen Seelen") (i. d. R. in der Kalligraphie eines Armeegenerals) bestehende Denkmäler waren bis 1945 überall auf öffentlichen Plätzen in Japan anzutreffen. Viele stammten aus der Zeit nach dem chinesisch-japanischen (1894/95) und dem russisch-japanischen Krieg (1904/05). Unter der Militaristenherrschaft wurde zwi-

I Hanrei-taimuzu Nr. 463 (Mai 1982), S. 76-89 (mit redaktioneller Vorbemerkung). Ein ausführliches Entscheidungsresumee (yoshi) des Gerichts war schon am Tage der Entscheidung in den Abendausgaben der Tageszeitungen veröffentlicht worden.

2 So H. Weber, Grundprobleme des Staatskirchenrats, JuS 1967, S. 433.

3 Die Liberaldemokratische Partei, die eine Politik der Re-Staatlichung des Yasukuni-Schreins in Tokyo, dem den Kriegsgefallenen gewidmeten nationalen Heiligtum, verfolgt (entsprechender Gesetzesentwurf wurde mehrfach im Parlament - bisher erfolglos - eingebracht), hat wenige Tage nach dem Urteilsspruch in einer "offiziellen Parteimeinung" die Entscheidung als "Angriff auf unsere traditionelle geistige Kultur" hef tig kritisiert. Vgl. Mainichi-Shimbun vom 30. 3. und Asahi-Shimbun vom 31. 3. 1982. 
schen 1939 bis zum Ende des Krieges systematisch in jedem Gemeindebezirk ein solches Chukonhi errichtet. Bei Betreten und Verlassen der Schule waren die im Sinne des "Tennō-Staats-Geschichtsbildes" erzogenen Schüler dazu angehalten, den von dem Denkmal repräsentierten Seelen der Gefallenen durch stilles Verharren und Verbeugen ihre Referenz zu erweisen. Nach dem Krieg wurde auf Anordnung des Alliierten Obersten Befehlshabers alle Kriegerdenkmäler (zusammen mit dem Staats-Shintō) als Ausdruck des Militarismus beseitigt (i. d. R. nur die aufgesetzte Inschriftenplatte, der Felssockel blieb stehen). ${ }^{4}$ Nachdem die Besatzungszeit Anfang 1952 zu Ende gegangen war, wurden viele dieser Chūkonhi auf Initiative der Hinterbliebenenvereinigungen erneut aufgestellt. So auch in der Stadt Minō. ${ }^{5}$ Das dortige Denkmal war ursprünglich im Jahre 1916 von der Ortsgruppe der landesweiten Veteranen-Vereinigung auf dem Schulhof der Grundschule errichtet worden. Es ist etwa sechs Meter hoch und drei Meter breit, wird von Bäumen umrahmt und von einer Steinballustrade umfaßt. Es ist den Seelen von 298 Kriegsgefallenen, deren Namenbrettchen sich im Inneren des Steins befinden, geweiht. Die Hinterbliebenenvereinigung hielt hier jährlich im April Gedenkzeremonien - abwechselnd in shintoistischem und buddhistischen Ritus - ab, an denen neben Vereinsmitgliedern auch der Bürgermeister und andere Gemeindebeamte als Gäste teilzunehmen pflegten und Beileidsworte verlasen. Die Bevölkerung nahm nicht teil.

Dieses Chūkonhi wurde 1975 nach Verhandlungen mit der Hinterbliebenen-Vereinigung von seinem Standort auf dem Schulgelände auf ein zu diesem Zweck von der Gemeinde erworbenen benachbarten Grundstück verlegt. Dabei entstanden Aufwendungen in Höhe von rund DM 788 200,- für den Grundstückskauf und in Höhe von rund DM 73 000,- für die Umsetzungsarbeiten. Eigentümer des Denkmals ist nicht die Stadt, sondern der Verein der Kriegshinterbliebenen, dem das von der Stadt erworbene Grundstück zum Zweck der Aufstellung des Denkmals unentgeltlich zur Verfügung gestellt worden war. ${ }^{6}$ Im Februar 1976 legten neun Bürgerinnen der Stadt Minō beim zuständigen LG Osaka gegen den Bürgermeister, den Erziehungsdezernenten und andere Gemeindebeamte Einwohnerklage (jümin soshō) gem. § 242,2 Lokal-Selbstverwaltungsgesetz $z^{7}$ wegen "unverantwortlicher Verwendung öffentlicher Gelder" ein und verlangten Rückerstattung eines Betrages i. H. von 35 Millionen Yen. Die Kläger vertreten die Ansicht, daß die Beklagten bei der Umsetzungsaktion gegen Art. 20 III und 89 der Verfassung verstoßen hätten.

$\mathrm{Zu}$ diesen Vorgängen und der vorangegangenen Etablierung des Staats-Shinto vgl. C. Coville, Shinto, Enginge of Government, The Transactions of the Asiatic Society of Japan, third Series, vol. 1 (1948), S. $1 \mathrm{ff}$. und S. Murakami, Japanese Religion in the Modern Century, (englisch) Tokyo 1980. Zur Entstehung des StaatsShinto grundlegend E. Lokowandt, Die rechtliche Entwicklung des Staats-Shinto in der ersten Hälfte der Meiji-Zeit (1868-1890), Studies in Oriental Religions, Bd. 3, Wiesbaden 1978.

5 Das Gericht weist ausdrücklich darauf hin, daß keine Klarheit darüber besteht, wer diese Wiedererrichtung finanziert habe.

6 Nach japanischem Recht bleibt das rechtliche Schicksal von Bauweken von dem des Grundstücks getrennt.

7 Jumin-sosho ist eine Art von Popularklage, mit der Bürger Akte der örtlichen Behörden angreifen können. Sie ist dem citizens' suit der amerikanischen Bundesstaaten nachgebildet. 
Art. 20 lautet:

"Die Freiheit des religiösen Bekenntnisses ist jedermann garantiert. Keine religiöse Gemeinschaft darf vom Staat mit Sonderrechten ausgestattet werden oder politische Macht ausüben. Niemand darf gezwungen werden, an religiösen Handlungen, Festen oder Veranstaltungen teilzunehmen.

Der Staat und seine Organe haben sich der religiösen Erziehung und jeder anderen Art religiöser Betätigung zu enthalten."

Art. 89 lautet:

"Offentliche Geldmittel und anderes öffentliches Vermögen dürfen zur Verwendung durch religiöse Organisationen oder Vereinigungen, zu deren Gunsten oder Erhaltung sowie für mildtätige, bildende oder wohltätige Werke, die nicht der öffentlichen Aufsicht unterstehen, weder ausgegeben noch zur Verfügung gestellt werden. ${ }^{8}$

Die Klägerinnen hoben besonders hervor, daß ihrer Ansicht nach die Stadtgemeinde einer religiösen Organisation, als welche sie die Vereinigung der Kriegshinterbliebenen klassifizierten, durch die Finanzierung der Denkmalverlegung und die kostenlose Nutzungsüberlassung des Grundstücks "Sonderrechte" eingeräumt habe.

Die Beklagten legten demgegenüber dar, daß das Denkmal nicht religiöser Natur sei. Auch die jährlich abwechselnd in shintoistischem und buddhistischem Ritus durchgeführten Gedenkzeremonien seien nicht als Religion, sondern als bloßes Brauchtum zu qualifizieren. Die notwendige Verlegung des Denkmals auf Kosten der Gemeinde habe daher keine verfassungsrechtlich relevante Dimension.

Beide Parteien hatten für ihre Auffassung Gutachten von Verfassungsrechts- und Religionswissenschaftlern eingeholt. ${ }^{9}$

\section{Die Entscheidung ${ }^{10}$}

Das Gericht gab der Klage statt und verurteilte die Beklagten zur Entrichtung eines Betrages in Höhe von rund DM 284,00 . Die Beklagten hätten es in rechtswidriger Weise unterlassen, gegenüber dem Ortsverein der Kriegshinterbliebenen die Beseitigung des Denkmals und die Räumung des städtischen Grundstücks und gegenüber dem Grundstücksverkäufer den Kaufpreis zurückzufordern. Alle im Zusammenhang mit der Umsetzung des Denkmals getroffenen Rechtshandlungen seien wegen Verfassungsverstoßes nichtig.

Das Gericht behandelt ausführlich Geschichte, Funktion und Zusammenhang mit dem (nationalen) Yasukuni-Schrein und den (lokalen) Gōkuku-jinja (den Schreinen zum 
Schutz des Staates) der Chūkonhi seit der Meiji-Restauration (1868) im allgemeinen sowie die konkreten Umstände des Minō-Denkmals im besonderen und gelangt zu der für seine Entscheidung grundlegenden Qualifikation des Denkmals als "religiöse Einrichtung". Die darauf bezogenen Handlungen der Stadt betrachtet es als "religiöse Betätigung" (Art. 20 III Verfassung), die Hinterbliebenen-Vereinigung als "religiöse Organisation oder Vereinigung" (Art. 89 Verfassung). Die entscheidungserheblichen Teile seiner Begründung lauten:

"Das Denkmal des vorliegenden Falles war von dem Ortsverein der Veteranenvereinigung zur Verehrung der Kriegsgefallenen von Minō ... errichtet worden. Es dient also der Ehrfurchtbezeugung gegenüber den im Krieg auf jammervolle Weise zu Tode gekommenen Soldaten und es wurde errichtet, die Seelen der Gefallenen zu trösten, die Leistungen, welche die Gefallenen zu Lebzeiten vollbracht haben, zu verherrlichen und sie nach ihrem gottverlassenen Tod als Götter zu verehren. Weil ferner die Denkmalinschrift 'chūkon die Seelen derjenigen bedeutet, die sich in Treue zum Tenno aufgeopf ert haben - die Aufschrift 'chū' (Loyalität) bedeutet die Pflicht der Untertanen gegenüber Staat und Tennō -, kann nicht abgestritten werden, daß hier der Tod auf dem Schlachtfeld gepriesen wird. ${ }^{11}$ Das Denkmal verlieh so den zahlreichen Zwischenfällen und dem Krieg als heiligem Krieg seit 1925 durch die Tennō-Herrschaft einen Sinn, was von der militaristischen Erziehung ausgebeutet wurde. Der Yasukuni-Schrein und die ihm untergeordneten Schreine zum Schutz des Staates fungierten bis zur Kriegsniederlage genauso wie die Chūkonhi. An Shintōfesten wurden alle Bürger einschließlich der Grundschüler gezwungen, an den Götterverehrungen teilzunehmen...

Das Chükonhi des vorliegenden Falles ist als ein Objekt eben einer solchen Verehrung eines dieser Chükonhi, eine Eigenschaft, die sich auch nach der Umsetzung nicht geändert hat. Das Denkmal des vorliegenden Falles ist durch eine (für Shintō-Schreine typische) Einfriedung umgeben, der Boden ist mit weißen Kieselsteinen ausgelegt, ... . es hat die gewaltige Höhe von 6,3 m. Eine derartige Struktur, ein derartiger Stil läßt die Atmosphäre eines unverletzlichen heiligen Bezirks aufkommen, wo gleich einem Schreinbezirk die Anwesenheit von Feierlichem und Mystischem empfunden wird. Im Chūkonhi des vorliegenden Falles . . . sind die Seelentäfelchen (reiji) als Gegenstände der Verehrung eingebaut, und die Hinterbliebenenfamilien haben angesichts dieses Denkmals ... die Vorstellung von der Existenz von etwas Ubernatürlichem ... Der Ortsverein Minō der Vereinigung der Hinterbliebenenfamilien hält vor dem Denkmal Gedenkzeremonien ... in [vom Gericht spezifisierten] shintoistischen oder buddhistischem Ritus ab...

Die Denk- und Erscheinungsformen von Religion sind höchst vielfältig und eine eindeutige Definition ist schwierig, jedoch kann man davon ausgehen, daß sich Religion aus der Uberzeugung von der Existenz von Úbernatürlichem, von die gewöhnliche Erkenntnis des Menschen Transzendierendem und der darauf sich gründenden Ehrfurcht bildet. Der Sinn der in Art. 20 und 89 der Verfassung erwähnten Religion muß auch als ein solches Phänomen verstanden werden . . . Somit handelt es sich bei dem Chükonhi des vorliegenden Falles ... um nichts anderes als um eine Tür religiöse Handlungen genutzte religiöse Einrichtung."

Von dieser Qualifikation des Denkmals ausgehend, kommt das Gericht zu dem Schluß, daß die Gebrauchsüberlassung des städtischen Grundstücks sowie die von der Stadt finanzierte Umsetzung verfassungswidrig seien. Es führt aus:

11 Weiter oben erwähnt das Urteil mit Blick auf die geltende (Friedens)-Verfassung (Präambel, Art. 9!), daß die Wertvorstellung von "chukon" von einer solchen des "Friedens" abgelöst worden sei, daher nur "Friedensmale" möglich seien. 
"Art. 20 I, S. 2 und III sowie Art. 89 der Verfassung inkorporieren den sog. Grundsatz der Trennung von Staat und Religion und beschränken sich nicht darauf, die Glaubensfreiheit des Volkes zu gewährleisten. Er ist dahingehend auszulegen, daß der Staat gegenüber sämtlichen Religionen sich neutral verhält und in dem Fall, daß er durch eine Handlung in eine Beziehung zur Religion tritt, ist unter Anschauung von Ziel und Wirkung dieser Beziehung zu prüfen, ob deren Ausmaß angesichts der verschiedenen sozio-kulturellen Voraussetzung unseres Landes und in Beziehung auf das fundamentale Ziel der institutionellen Sicherung der Gewährleistung der Glaubensfreiheit des Volkes die Grenzen des Angemessenen überschreitet (in welchem Falle eine solche Beziehung verboten ist). (Vgl. OGH vom 13.7. 1977, Minshū Bd. 31, Nr. 4, S. 533). ${ }^{12}$

"Religiöse Organisationen oder Vereinigungen" in Art. 89 und "religiöse Betätigung" in Art. 20 III der Verfassung seien nim Hinblick auf Bedeutung und Funktion eines solchen Prinzips der Trennung von Staat und Religion" in strengem Sinne zu interpretieren; die genannten Verfassungsworte bezögen sich auf alle Arten religiöser Kulthandlungen. Eine solche weite Interpretation sei unter den spezifisch japanischen Bedingungen besonders angebracht:

"Der Charakter unseres Volkes ist hinsichtlich der Religion äußerst ungebunden (unbeständig, treulos: musessō), es ist insofern ein eigentümliches Volk, als es keinen Unterschied zwischen Gott (kami) und Mensch macht. (So hat z. B. der Tennō am 1. Jan. 1946 ein Dekret "Die Verkündung der Menschlichkeit des Tennō" erlassen). Zur Verwurzelung des von der neuen Verfassung angenommenen Prinzips der Trennung von Staat und Religion in einer solchen Gesellschaft, hat man dieses Prinzip streng zu interpretieren, und wird es nicht durchgesetzt, so wird es zunichte ..."

Das Gericht kommt zu dem Schluß, daß die kostenlose Gebrauchsüberlassung des städtischen Grundstücks an die Hinterbliebenen-Vereinigung sowie die durch die Stadt finanzierte Umsetzung des Denkmals der "Erhaltung der religiösen Zwecke" des Denkmals gedient habe, womit die Stadt "nichts anderes getan hat, als religiösen Aktivitäten Unterstützung und Förderung zuteil werden zu lassen“. Im Gegensatz zum Streitgegenstand des bisher vom OGH zur Auslegung des Trennungsprinzips entschiedenen Falles, ${ }^{13}$ sei im vorliegenden Fall eine höchst intensive und langanhaltende Beziehung zwischen der Stadt und dem Chūkonhi zu konstatieren.

\section{Bisherige Rechtsprechung zum Trennungsprinzip}

Die Entscheidung des LG Osaka, die ein Kriegsgefallenendenkmal als "religiöse Einrichtung", darauf bezogene städtische Handlungen als von der Verfassung untersagte "religiöse Betätigung" einer öffentlichen Körperschaft ansieht, ist in einen Zusammenhang mit der bisherigen Rechtssprechung zur Auslegung des Trennungsprinzips zu stellen.

$12 \mathrm{Zu}$ dieser Bezugnahme auf das bislang einzige einschlägige Erkenntnis des OGH vgl. weiter unten.

13 Dazu unten. 
Einschlägig ist hier im wesentlichen nur die durch drei Instanzen geführte Auseinandersetzung um die Verfassungsmäßigkeit einer nach shintoistischem Ritus durchgeführten Grundsteinlegungsfeier in einer öffentlichen Schule. Im Jahre 1965 war die Stadtverwaltung von Tsu in der Provinz Mie von einem Stadtratsmitglied auf Rückzahlung von rund DM 80,-, die dem Shintopriester, der die Zeremonie durchgeführt hatte, aus der Stadtkasse gezahlt worden war, verklagt worden. Das LG Tsu klassifizierte die Grundsteinlegungszeremonie nicht als religiöse Handlung, sondern als bloßes Brauchtum und wies daher die Klage ab. Das OLG Nagoya hob dieses Urteil auf und gab der Klage statt, weil der religiöse Charakter weder der Zeremonie noch des Shintō als solchem ${ }^{14}$ geleugnet werden können. ${ }^{15}$ In seiner ersten Entscheidung zu diesem Problembereich hob der OGH mit Mehrheit von zehn gegen fünf das Urteil der zweiten Instanz auf und bestätigte im Ergebnis das erstinstanzliche Urteil. ${ }^{16}$ Von Bedeutung ist die pragmatische Sicht, mit der sich das Gericht der Interpretation des Trennungsprinzips näherte. Es wies auf die mannigfaltigen Konzequenzen, die eine strikt durchgeführte Trennung von Religion und Staat mit sich brächte. So könnte die (sehr erhebliche) staatliche Unterstützung von Privatschulen in klerikaler Trägerschaft ebenso zweifelhaft werden wie Maßnahmen des Denkmalschutzes (Tempel, buddhistische Statuen etc.) und das Tätigsein religiösen Personals in den Strafvollzugsanstalten. Das Gericht vertrat die Auffassung, daß Religion nicht lediglich eine individuelle Angelegenheit sei; Religion manifestiere sich vielmehr auch in zahlreichen Bereichen der gesellschaftlichen Sphäre. So hätten historische Entwicklungen in Bereichen wie Erziehung, Wohlfahrt, Kultur und Volksbräuchen zu mannigfaltigen religiösen Implikationen geführt, die den Staat nicht hindern dürften, seine Politik der Förderung von Bildung, Wohlfahrt, Kultur etc. durchzuführen. Eine rein mechanische Trennung von Staat und Religion sei also "so gut wie unmöglich." Die Verfassung enthalte insofern ein Ideal, das den Staat hindere, die garantierte Religionsfreiheit durch Favorisierung einer bestimmten Religion zu verletzen. Das Trennungsprinzip beinhalte lediglich die religiöse Neutralität des Staates, Beziehungen zwischen Staat und Religion seien nicht völlig verboten. "Vielmehr ist bei einer Handlung, die eine Beziehung zur Religion herbeiführt, unter Berücksichtigung ihres Ziels und ihrer Wirkung zu prüfen, ob diese Beziehung unter den oben genannten Bedingungen (Notwendigkeit des Staates, religiöse Phänomene zur Kenntnis zu nehmen etc.) die Grenze des Angemessenen überschreitet; in diesem Fall ist sie verboten. ${ }^{17}$

14 Unter der Meiji-Verfassung (von 1889) war zwar in Art. 28 die Religionsfreiheit garantiert. Da Shinto aber nicht als Religion klassifiziert wurde, konnte Art. 28 nicht gegen die aus dem Staats-Shinto sich ergebenden Beamten- und Bürgerpflichten geltend gemacht werden.

15 Eine Ubersetzung der wesentlichen Teile dieses umfangreichen Erkenntnisses bei E. Lokowandt, Zum Verhältnis von Staat und Shinto im heutigen Japan. Eine Materialsammlung, Wiesbaden 1981, S. 89 ff. Vgl. ferner H. Tanaka, The Japanese Legal System, Tokyo 1976, S. 735 f.

16 Entscheidung des Großen Senats vom 13. 7. 1977, in Hanrei Jiho Nr. 855, S. 24 ff. Ubersetzung bei Lokowandt (Anm. 15), S. 145 ff. Ausführliche Rezension in Law in Japan, Bd. 10 (1977), S. $161 \mathrm{ff}$.

$17 \mathrm{Daß}$ das Gericht dann weiter argumentiert, die fragliche Grundsteinlegungszeremonie (die es neutral "kikoshiki", d. z. "Zeremonie zum Beginn der Bauarbeiten" und nicht wie die Vorinstanz "jichin-sai", d. h. "Reinigung des Baugrundstückes" nennt) habe ihren ursprünglichen religiösen Charakter im Lauf der Zeit völlig verloren, ist von diesem Ansatz her überflüssig und inkonsequent und in Sache höchst fragwürdig. 
Demgegenüber berief sich die Minderheit in einem gemeinsamen Votum und mit Nachdruck der damalige (christliche) Gerichtspräsident in seinem Sondervotum auf die Interpretation des ersten Amendments der USA-Verfassung durch den Supreme Court, nach der "Religion" und "religiösen Begriffen" in der Verfassung der USA "eine weitest mögliche Interpretation zuteilwerden soll."

In Auseinandersetzung mit dieser Rechtssprechung betont das LG Osaka, daßes sich im Falle der Grundsteinlegungszeremonie um einen einmaligen Vorgang gehandelt habe, im Falle des Chūkonhi aber "die Verbindung zwischen Stadt und Chūkonhi äußerst stark" gewesen sei. Die Stadt habe ein "enormes Maß an Gleichgültigkeit gegenüber dem Prinzip der Trennung von Staat und Religion an den Tag gelegt. «18

\section{Würdigung}

Es sind zwei Fragen, die in der bisherigen Rechtsprechung zum Prinzip der Trennung von Staat und Religion zu beantworten waren und in Zukunft weiter zu beantworten sein werden:

(1) Sind die das Trennungsprinzip normierenden Verfassungsbestimmungen überhaupt tangiert, handelt es sich bei den fraglichen staatlichen Handlungen um solche religiöser Natur?

(2) Wird diese Frage im Sinne des Vorliegens einer religiös relevanten staatlichen Handlung beantwortet, ist der Regelungsbereich des Trennungsprinzips also betroffen, so ist nach dem Inhalt dieses Regelungsbereichs also danach zu fragen, wie das in der japanischen Verfassung inkorporierte Trennungsprinzip aufzufassen ist.

Im Bereich der ersten Frage geht es um eine Abgrenzung von Religion und Brauchtum. Die hier geforderte Unterscheidung dürfte aber in dem in aller Regel allein in Betracht kommenden Grenzbereich nach allen denkbaren - historischen, soziologischen, psychologischen - Betrachtungsweisen selten klar zu treffen sein. Im Zweifel wird man sich ohnehin im Dienste eines weitestmöglichen Schutzes der Religionsfreiheit für das Vorliegen einer "religiösen" Handlung entscheiden müssen. In den entschiedenen Fällen (Grundsteinlegung und Chūkonhi) war dies schon wegen der durchgeführten eindeutig shintoistischen und buddhistischen Riten ohne große Bedenken möglich und jedenfalls eher vertretbar als dies eine Entscheidung zugunsten bloßen Brauchtums gewesen wäre.

Wird daher in diesem Grenzbereich die Abwägung eher zugunsten von Religion erfolgen müssen, so tritt die Reflexion der zweiten Frage in den Vordergrund der richterlichen Entscheidungsfindung. Das OLG Nagoya faßte das Trennungsprinzip der Verfassung im Sinne einer strikten Trennung, im Sinne eines Verbots staatlicher Zurkenntnisnahme religiöser Phänomene schlechthin, auf. ${ }^{19}$ Es führte aus:

18 Das Gericht weist ferner darauf hin, daß die von der Stadt Hiroshima vor dem Denkmal für die Opfer der Atombombe abgehaltenen Gedenkfeiern in "nicht-religiösen Formen " durchgeführt würden.

19 Kommentare und Lehrbücher entwickeln hier keine substantiierten Auffassungen. Die im Gefolge der OGHEntscheidung vom 13.7. 1977 entstandene Auf satzliteratur beschränkt sich weitgehend auf ein Referieren 
"Um das Prinzip der Trennung von Staat und Religion in Japan richtig verstehen zu können, muß man sich in Erinnerung rufen, daß es unmißverständlich in die Verfassung eingeführt worden ist, im Zusammenhang mit den mannigf altigen Uebeln, zu denen während des Krieges und schon zuvor die Verbindung von Schrein-Shinto und Staatsgewalt geführt hatte . . A A diesem Grund erließ das Oberkommando der Alliierten Streitkräfte am 15. Dezember 1975 das 'Memorandum bezüglich der Abschaffung der Garantie, Unterstützung, Erhaltung, Aufsicht und Verbreitung des Staats-Shintō (Schrein-Shintō) durch die Regierung'. Durch dieses Memorandum wurde die vollständige Trennung von Staat und Schrein-Shintō angeordnet und eindeutig bestimmt, daß der Schrein-Shintō als eine Religion neben anderen auf der gleichen rechtlichen Grundlage wie alle anderen Religionen zu stehen habe und daß dazu alle Religionen, einschließlich des Shintō, vom Staat zu trennen seien ... Hier wurde - mit dem Hauptziel der Auflösung des Staats-Shint $\boldsymbol{\delta}$ - der Weg zu einer durchgreifenden Trennung von Staat und Religion, zu einer konsequenten Trennung von Staat und Religion eröffnet . . . beinhaltete diese Shintō-Direktive nicht nur eine Trennung von Staat und Kirche, wie sie in den Ländern Europas gehandhabt wird, sondern, durchgreifender, eine Trennung von Staat und Religion ... Man kann sagen, daß das Spezifische des Prinzips der Trennung von Staat und Religion in Japan eben darin liegt, daß die geistige Herrschaft des Staats-Shintō der Kriegs- und Vorkriegszeit durch die Verfassung vollständig beseitigt und auf diesem Wege Religionsfreiheit errichtet und garantiert wurde. Hinzu kommt, daß in Japan, anders als in den christlichen Ländern des Westens, niemals in der Geschichte eine einzelne Religion eine beherrschende Stellung eingenommen hat. Als Besonderheit der religiösen Verhältnisse ist anzuführen, daß, während Volk und Sprache einheitlich waren, verschiedene Religionen pluralistisch nebeneinander bestanden und sich nebeneinander entwickelten. Daher ist festzustellen: in einem Land, in dem wie in Japan Religionen pluralistisch nebeneinander bestehen, ist es erst dann möglich, die Religionsfreiheit zu garantieren, wenn man, um die Verbindung des Staats mit einer einzelnen Religion auszuschließen, eine durchgreifende Trennung von Staat und Religion einführt."

Das japanische Trennungsprinzip wird dann abgegrenzt von dem - etwa unter dem Grundgesetz der Bundesrepublik Deutschland bestehenden - System religiöser Neutralität und verglichen mit dem US-amerikanischen System nach der Judikatur zum ersten Zusatzartikel der Verfassung.

Der OGH gewann in seiner Entscheidung über die Grundsteinlegungszeremonie insofern Neuland, als zum ersten Mal die Grenzen und Alternativen einer Interpretation des Trennungsprinzips aufzuweisen versucht, dabei "religiöse Neutralität" des Staates einem strikten Trennungssystem gegenübergestellt wurde:20

"Allgemein versteht man unter dem Prinzip der Trennung von Staat und Religion den nicht-religiösen Charakter des Staates bzw. seine religiöse Neutralität. Begründet wird es damit, daß Fragen der Religion oder ihrem Wesen nach Fragen des Gewissens des einzelnen seien . . . und

dieser Entscheidung. Etwa: I. Sato, in: Hundert Entscheidungen in Religionsangelegenheiten, (Sonderheft "Jurist" No. 37, Juli 1972, S. 32-35; N. Kobayashi, in: Horitsu-jiho Bd. 49, Nr. 11, S. 89-96; H. Wada, in: Hogaku-seminar 1979/9, S. 2-9; S. Mori, in: Hundert verfassungsrechtliche Entscheidungen Bd. 1 (Sonderheft "Jurist" No. 68, April 1980), S. 48 f. mit weiteren Nachweisen. Letzterer unterscheidet eine "Lehre der vollständigen Trennung" von einer "Lehre der begrenzten Trennung".

20 Ähnlich der Auslegung von Art. 140 GG durch Maunz in Maunz-Dürig-Herzog-Scholz, GG-Kommentar, Art. 140, RN $43 \mathrm{ff}$., besonders $52 \mathrm{ff}$., allerdings mit der grundlegenden Einschränkung, daß der japanische OGH von der vollständigen Trennung als einem "Ideal" spricht, das lediglich (derzeitig jedenfalls) nicht voll realisiert werden kann, während die Konstatierung eines solchen Ideals im System des deutschen Staatskirchenrechts wohl als verfassungswidrig anzusehen wäre. 
daß daher der Staat, der ja eine weltliche Gewalt ist, Fragen der Religion außerhalb des Bereichs der öf fentlichen Gewalt ansiedeln müsse und sich in Fragen der Religion nicht einmischen dürfe. Selbstverständlich bestehen im Verhältnis von Staat und Religion je nach den historischen und gesellschaftlichen Bedingungen eines Landes Unterschiede ... Anders als etwa in den christlichen oder islamischen Staaten ist die religiöse Lage in Japan pluralistisch . . . Um in einer solchen religiösen Lage die Glaubensfreiheit zu sichern, reichte es nicht aus, einfach nur die Glaubensfreiheit ohne Einschränkung zu garantieren. Darüber hinaus war es notwendig, auch Bestimmungen zur Trennung von Staat und Religion aufzunehmen, um eine Verbindung des Staates mit welcher Religion auch immer auszuschließen. Hieraus folgt, daß die Verfassung, als sie die Bestimmungen zur Trennung von Staat und Religion aufnahm, das Ideal einer vollkommenen Trennung von Staat und Religion verfolgte, daß sie dadurch den nicht-religiösen Charakter des Staates bzw. seine religiöse Neutralität sicher verankern wollte. Die Bestimmungen zur Trennung von Staat und Religion beinhalten ihrem Wesen nach jedoch eine institutionelle Garantie, sie garantieren nicht direkt die Glaubensfreiheit als solche. Vielmehr wollen sie . . die Garantie der Glaubensfreiheit indirekt weiter sichern. Religion umfaßt jedoch nicht nur den persönlichen Glauben des einzelnen, gewöhnlich weist sie zugleich auch eine äußerst vielfältige äußere, gesellschaftliche Seite auf, die in weiten Bereichen, in Erziehung, Wohlfahrt, Kultur, Volkssitte usw. mit dem Leben der Gesellschaft in Berührung kommt. Hieraus folgt dann zwangsläufig, daß der Staat es gar nicht vermeiden kann, daß eine Beziehung zu ihm und der Religion entsteht, wenn er das Leben der Gesellschaft Regelungen unterwirft oder wenn er Maßnahmen zur Unterstützung oder Förderung von Erziehung, Wohlfahrt, Kultur usw. durchführt. Folglich muß festgestellt werden, daß es im realen Sinne nahezu unmöglich ist, eine vollständige Trennung von Staat und Religion zu verwirklichen. Wie hieraus zu ersehen ist, ist auch die in der Verfassung garantierte Trennung von Staat und Religion nicht ohne ihre eigenen, ihr immanenten Grenzen. Bei einer Umsetzung des Prinzips der Trennung von Staat und Religion in die reale Staatswirklichkeit ist somit . . . im Zusammenhang mit dem grundlegenden Ziel dieser Institution, der Sicherung der Garantie der Glaubensfreiheit, die Frage zu prüfen, in welchen Fällen und in welchem Umfang diese Beziehungen die Grenzen des Zulässigen überschreiten. Deshalb ist der Grundsatz der Trennung von Staat und Religion . . . so auszulegen, daß er zwar religiöse Neutralität des Staates fordert, Beziehungen zwischen Staat und Religion aber nicht vollkommen verbietet. Vielmehr ist bei einer Handlung, die eine Beziehung zur Religion herbeiführt, unter Berücksichtigung ihres Ziels und ihrer Wirkung zu prüfen, ob diese Beziehung unter den oben genannten Bedingungen die Grenzen des Angemessenen überschreitet; in diesem Falle ist sie verboten. (Solche das Maß des Angemessenen überschreitende Handlungen sind solche), die ein Ziel von religiöser Bedeutung verfolgen oder die in ihrer Wirkung auf eine Hilfe, Unterstützung, Förderung bzw. Unterdrückung, Einmischung usw. einer Religion hinauslaufen . .."

Methodisch unterscheiden sich beide Auslegungen nicht im grundsätzlichen Blickwinkel - beiden geht es um eine verobjektivierende Konkretisierung des Trennungsprinzips vornehmlich unter Anschauung der in die Verfassung eingegangenen soziohistorischen Prägefaktoren - als vielmehr darin, daß die vom OLG Nagoya vertretene "Lehre der völligen Trennung" (so auch die Mindermeinung im OGH-Urteil) es versäumt hat, Bewußtsein zu entwickeln für die immanenten Grenzen auch des verfassungsrechtlichen Trennungsgrundsatzes. Das LG Osaka, des sicher stark zur strikten Trennung tendiert, hat diese nichtdestoweniger an dem vom OGH aufgerichteten Maßstab angebunden und so seiner Entscheidung eine nur schwer anfechtbare Folgerichtigkeit verliehen. 